\title{
Thoroughbred Race Horses Management: A Key Role of the Veterinarian
}

\author{
Adolfo Godoy Pinto* \\ Department of Veterinary Medicine and Animal Science, University of Chile, Chile \\ ${ }^{\star}$ Corresponding author: Adolfo Godoy Pinto, Professor, Veterinary Medicine and Animal Science, University \\ of Chile, Chile; E-mail: agodoy@uchile.cl
}

Citation: Adolfo Godoy Pinto (2013) Thoroughbred Race Horses Management: A Key Role of the Veterinarian. J Vet Sci Anim Husb 1(1): e102. doi: 10.15744/2348-9790.1.e102

Received Date: July 03, 2013 Accepted Date: July 29, 2013 Published Date: August 01, 2013

\begin{abstract}
The veterinarian must confront many situations when working with thoroughbred horses in which he must solve and make decisions that lead to balance between the animal and trainer / owner of the horse, the above should be resolved in an ethical and professional manner.
\end{abstract}

The management and demand levels to which thoroughbred horses are subjected leads to an organic stress reaction absolutely necessary to maintain homeostasis with an appropriate allostatic load depending the systemic demand they undergo. However, if these conditions are inadequate such as, insufficient training, overtraining, increased levels of competition in distance and frequency, stressful situation becomes a chronic condition that seriously compromises the animal's homeostasis thereby acting as a predisposing factor that could trigger in some cases a wide range of diseases that may compromise the performance and the life of the animal in severe cases (Infectious diseases, upper airway diseases, musculoskeletal diseases, among others). Although catecholamines and cortisol have a positive effect on the immune system by promoting migration and maturation of immuno competent cells, these substances when are chronically sustained over time at high levels produces an immunosuppressive response. Briefly, the glucocorticosteroids (cortisol) acts predominantly on type II cell receptors which results in stimulation of anti-inflammatory protein synthesis and decreases the maturation and migration of immune cells. Additionally, the chronic levels of catecholamines significantly modify the behavior of leukogram which also shows decreased immune protection. It is known that chronic stress inhibits the humoral and cellular immune response, decreases the proliferation, migration and $\mathrm{T}$ cell cytotoxicity, reduced antibody production, reduce the synthesis of proinflammatory cytokines and interleukins etc. This may be prevented by a rational and appropriate use of animals in competition which although it is dependent on a great number of variables an important factor is the role played by veterinarians who have the responsibility to authorize or recommend animal for competitions.

We have observed in racehorses subjected to an appropriate level of competence that the packed cell volume (PCV) and plasma catecholamines, cortisol and glucose in blood samples obtained after competition are higher than basal values.
The catecholamines increased 6-7 times while cortisol was enhanced by $25 \%$. However samples withdrawn 24 hours after the situation of stress caused by competition was not possible to find values significantly different from basal values, indicating that the deleterious effects of stress occur when this is in a chronic way. In the author's experience those animals of low handicap that usually undergo more than one competition per week, or who frequently travel between different racetracks to compete have a higher prevalence of diseases such as pharyngeal lymphoid hyperplasia (young animals), pleuropneumonia, cellulitis and even increases the prevalence of dermatophytosis, which is not observed with the same prevalence in animals handled properly.

On the other hand, evaluation and clearance for competition of horses is not the only function that the veterinarian must accomplish also the vet must perform a complete physical evaluation including respiratory and musculoskeletal systems. Additionally, proper evaluation and interpretation of cardiovascular examination in thoroughbred horses is particularly important because these animals often have high incidence of signs such as murmurs, abnormal heart sounds, arrhythmias, among others, many of which are only the expression of cardiovascular adaptation to exercise, however, if only one of these animals present any of these signs coexisting with a pathological heart disease every effort to differentiate between those animals is justified. Having said that, the veterinarian has a big responsibility that should be imposed against the interests of owners and /or trainers and a complete cardiovascular evaluation prior to the competition is always needed.

To the author's judgment, the veterinarian plays an important role in avoid situations that may jeopardize the life and/ or health of these animals and therefore requires an ethical, competent and responsible behavior where the welfare of animals should be imposed on all types of interests.

After many years of practice as a veterinarian at the clinic for horses I can conclude that the best professional is not the most knowledgeable about a particular subject, or the one that best handles a procedure, nor the best educated, or the most experienced. The best professional is the one who has all these features and the ability to combine them to manage and deal with 
new situations. I mean, is the one able to act in an appropriate manner, quality and humanity facing those problems that apply to the profession, selecting and mobilizing various internal and external resources and at the same time, being able to give reasons for their decisions and taking charge of their results, that is one who is able to act with responsibility. Finally, it is important to consider that in the exercise of the profession is our obligation to give the best living conditions to horses so that they can fulfill their role to entertain without postponing or sacrificing welfare conditions. Also not only are the horses involved in competition but also to some extent we are responsible for the lives of human beings, as in the case of the jockeys or riders who rely on our work and that the decision to authorize the participation of an animal in competition, has been done properly from professional standpoint and ethics.

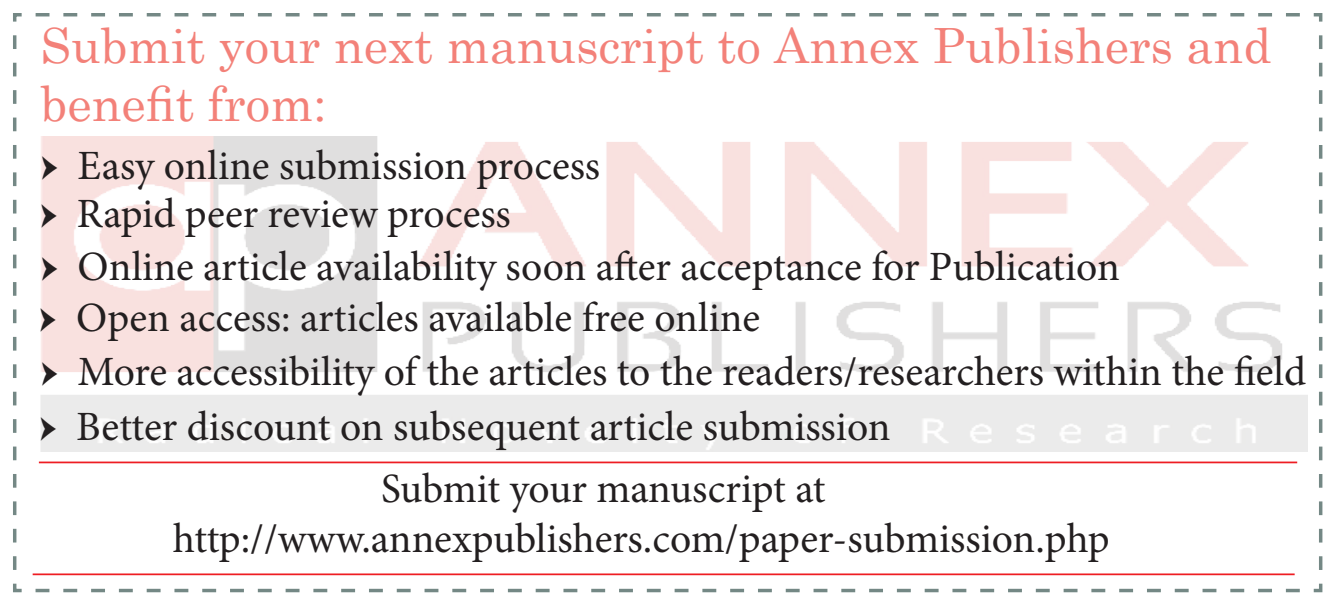

